\title{
Long non-coding RNA HAND2-AS1 targets glucose metabolism and inhibits cancer cell proliferation in osteosarcoma
}

\author{
SHUNGUANG CHEN*, XIAOMING XU*, SHENGJUN LU and BIAO HU \\ Department of Orthopedics, Jingzhou Central Hospital, Jingzhou, Hubei 434020, P.R. China
}

Received June 2, 2018; Accepted April 25, 2019

DOI: $10.3892 / \mathrm{ol} .2019 .10445$

\begin{abstract}
Long non-coding RNA heart and neural crest derivatives expressed 2-antisense RNA 1 (lncRNA HAND2-AS1) is a known tumor suppressor gene in endometrioid endometrial carcinoma; however, its function in osteosarcoma is currently unknown. In the present study, HAND2-AS1 expression in the tumor tissues and adjacent healthy tissues of patients with osteosarcoma, and in the serum of patients and heathy controls was detected by reverse transcription-quantitative polymerase chain reaction. IncRNA HAND2-AS1 small interfering RNA was transfected into osteosarcoma cells, and cell proliferation, glucose transporter 1 (GLUT1) expression and glucose uptake were detected using the Cell Counting Kit-8, western blotting and glucose uptake assays, respectively. The results revealed that the expression levels of HAND2-AS1 were reduced in cancer tissues compared with those in healthy tissues. Levels of HAND2-AS1 were also reduced in the serum of patients with osteosarcoma compared with those of the control subjects. A significant association was observed between serum levels of HAND2-AS1 and tumor size, but not tumor metastasis. HAND2-AS1-knockdown promoted osteosarcoma cell proliferation, increased glucose uptake and upregulated GLUT1 expression. It was therefore concluded that lncRNA HAND2-AS1 may inhibit the proliferation of osteosarcoma cells by targeting glucose metabolism.
\end{abstract}

\section{Introduction}

As a malignancy that develops in the bone, osteosarcoma primarily affects children, adolescents and young adults (1). Although the incidence is low, osteosarcoma is one of the leading causes of cancer-associated mortality among teenagers

Correspondence to: Dr Shunguang Chen, Department of Orthopedics, Jingzhou Central Hospital, 60 Jing Zhong Road, Jingzhou, Hubei 434020, P.R. China

E-mail: ydjngg2@163.com

*Contributed equally

Key words: long non-coding RNA HAND2-AS1, osteosarcoma, glucose uptake, glucose transporter 1
(13-19 years old) and young adults (20-30 years old) worldwide (2). With the developmement and application of systemic chemotherapy, the survival rate of patients with osteosarcoma has improved significantly (3). However, this is challenged by the high prevalence of tumor metastasis by the time of diagnosis, and despite appropriate treatment, the 5-year surival rate of patients with distant tumor metastasis remains poor $(\geq 20 \%$ ) (4). Therefore, early diagnosis and treatment are prominent factors in the surival of patients with osteosarcoma. Unclear pathogenesis is a principal cause of treatment failure in osteosarcoma (3), and in-depth investigation of the mechanisms of development and progression of osteosarcoma may improve its diagnosis and treatment.

It has been demonstrated that the development and progression of osteosarcoma is frequently associated with the altered expression of specific long non-coding RNAs (lncRNAs) (5), indicating the invovlement of lncRNA in disease pathogenesis. Glucose metabolism serves a pivotal role in cancer growth by providing energy for survival and cellular proliferation (6). lncRNA heart and neural crest derivatives expressed 2-antisense RNA 1 (HAND2-AS1) is a recently identified lncRNA that serves as a tumor suppressor gene in endometrioid endometrial carcinoma by inhibiting the invasion and migration of cancer cells (7). However, its involvement in other cancer types is unknown. It has been reported that certain lncRNAs may interfere with glucose metabolism in cancer cells, promoting or inhibiting growth, development and progression (8). In the present study, HAND2-AS1 was downregulated in osteosarcoma, where its influence on glucose metabolism may regulate tumor growth. This revealed a novel function for HAND2-AS1 and provides a potential therapeutic target for osteosarcoma.

\section{Materials and methods}

Subjects. A total of 48 patients with osteosarcoma were recruited. Patients were diagnosed by pathological examination and treated in Jingzhou Central Hospital (Jingzhou, Hubei, China) between January 2015 and January 2017. Inclusion criteria were as follows: i) Osteosarcoma confirmed by pathological examinations; ii) patients who were willing to participate; and iii) patients who received surgical resection. Exclusion criteria were as follows: i) Patients suffering from other types of malignancies; ii) patients with other types of severe disease, such as metabolic diseases and severe infections; and iii) patients who were treated in other hospitals prior 
to the study. The cohort included 28 males and 20 females (age range, $12-67$ years; mean age, $32 \pm 8.5$ years), and 44 healthy volunteers were recruited as controls. The control group included 26 males and 18 females (age range, 14-66 years; mean age, $34 \pm 7.9$ years). No significant differences in age and sex were present between the two groups. All patients provided written informed consent.

Specimen collection and processing. Tumor tissues and adjacent healthy tissues (within a $2-\mathrm{cm}$ area around the tumor) were collected during surgical resection, and confirmed by pathological examination. On the day of admission, blood was extracted from the elbow vein of each of the patients and healthy controls. The blood was stored at room temperature for $2 \mathrm{~h}$, followed by centrifugation at $1,250 \mathrm{x} \mathrm{g}$ at room temperature for $20 \mathrm{~min}$ for serum. All specimens were stored in liquid nitrogen for long-term use.

Cell lines and transfection. The human osteosarcoma MG-63 and SAOS-2 cell lines, and the normal bone hFOB cell line were obtained from the American Type Culture Collection (ATCC). The MG-63 and SAOS-2 cells were cultured in Eagle's minimum essential medium (cat. no. 30-2003; ATCC) supplemented with $10 \%$ fetal bovine serum (FBS; Thermo Fisher Scientific, Inc.). hFOB cells were cultured in McCoy's 5a modified medium (cat. no. 30-2007; ATCC) containing 10\% FBS. All cells were cultured at $37^{\circ} \mathrm{C}$ with $5 \% \mathrm{CO}_{2}$. HAND2-AS1 small interfering RNA (siRNA; 5'-CCGAGGUGCUCCAAU AUUATT-3') and negative control siRNA (5'-UUCUCCGAA CGUGUCACGUdTdT-3'); were purchased from Shanghai GenePharma Co., Ltd. All three cell lines were cultured to $80-90 \%$ confluence, and $5 \times 10^{5}$ cells/sample were transfected with $50 \mathrm{nM}$ siRNA using Lipofectamine ${ }^{\circledR} 2000$ reagent (cat. no. 11668-019; Invitrogen; Thermo Fisher Scientific, Inc.). Reverse transcription-quantitative polymerase chain reaction (RT-qPCR) was performed to measure the expression levels of HAND2-AS1, and to confirm a $<50 \%$ reduction prior to subsequent experiments. Subsequent experiments were performed at $24 \mathrm{~h}$ after transfections. The control group for transfection was un-transfected cells, and the negative control group was negative control siRNA-transfected cells.

Glucose uptake assay. A total of $5 \times 10^{5}$ cells were cultured in each well of a 6 -well plate. Cells were incubated for $24 \mathrm{~h}$ and washed twice with PBS. Glucose uptake was initiated by incubating cells with Krebs-Ringer-HEPES (KRH) buffer [120 mM NaCl, $25 \mathrm{mM}$ HEPES (pH 7.4), $1.2 \mathrm{mM} \mathrm{MgSO}_{4}$, $1.3 \mathrm{mM} \mathrm{CaCl}_{2}, 5 \mathrm{mM} \mathrm{KCl}$ and $\left.1.3 \mathrm{mM} \mathrm{KH}_{2} \mathrm{PO}_{4}\right]$ containing $1 \mu \mathrm{Ci}[3 \mathrm{H}]-2$-deoxyglucose (PerkinElmer, Inc.) for $25 \mathrm{~min}$ at $37^{\circ} \mathrm{C}$. Cells were washed twice with ice-cold KRH buffer to halt glucose uptake, and lysed using lysis buffer [10 $\mathrm{mM}$ Tris-HCl (pH 8.0) and 0.2\% SDS]. Radioactivity was measured using liquid scintillation spectrometry, and glucose uptake was presented as disintegrations per min. The experiment was performed in triplicate.

$R T$ - $q P C R$.TRIzol ${ }^{\oplus}$ reagent was used to extract the total RNA from tumor tissues, adjacent healthy tissues and the 3 cell lines, MG-63, SAOS-2 and hFOB. The RNA concentration was measured using a NanoDrop ${ }^{\mathrm{TM}} 2000$ Spectrophotometer (Thermo Fisher

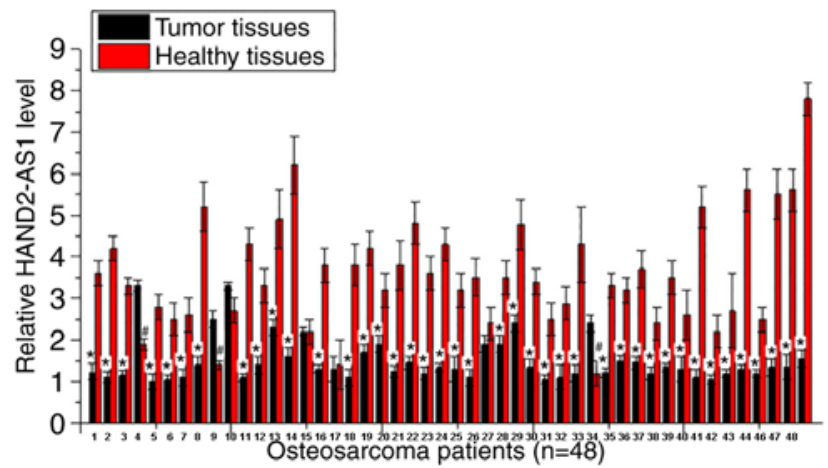

Figure 1. Expression of HAND2-AS1 in tumor tissues and adjacent healthy tissues of 48 patients with osteosarcoma. Expression levels of HAND2-AS1 in osteosarcoma tissues and healthy tissues of 48 patients were measured using reverse transcription-quantitative polymerase chain reaction. The expression level of HAND2-AS1 was significantly lower in osteosarcoma tissues compared with that in adjacent tissues in the majority of patients with osteosarcoma. ${ }^{*} \mathrm{P}<0.05$ compared with adjacent tissues, ${ }^{\#} \mathrm{P}<0.05$ compared with osteosarcoma tissues. $n=3$. HAND2-AS1, heart and neural crest derivatives expressed 2-antisense RNA 1.

Scientific, Inc.), and samples with an A260/A280 ratio between 1.8 and 2.0 were reverse transcribed. The PrimeScript RT reagent kit (Takara Bio, Inc.) was used to perform reverse transcription reactions with poly(T) as the primer. Reaction conditions were: $25^{\circ} \mathrm{C}$ for $6 \mathrm{~min}, 54^{\circ} \mathrm{C}$ for $20 \mathrm{~min}$ and $80^{\circ} \mathrm{C}$ for $5 \mathrm{~min}$. The $\mathrm{SYBR}^{\circledR}$ Green Real-Time PCR Master mix (Thermo Fisher Scientific, Inc.) was used to prepare the PCR. The sequences of primers were as follows: HAND2-AS1 forward, 5'-GGGTGTTTACGT AGACCAGAACC-3' and reverse, 5'-CTTCCAAAAGCCTTC TGCCTTAG-3'; and $\beta$-actin forward, 5'-GACCTCTATGCC AACACAGT-3' and reverse, 5'-AGTACTTGCGCTCAGGAG GA-3'. PCR was conducted using the CFX96 Touch $^{\text {TM }}$ Real-Time PCR Detection system (Bio-Rad Laboratories Inc.). PCR conditions were as follows: $95^{\circ} \mathrm{C}$ for $40 \mathrm{sec}$, followed by 40 cycles at $95^{\circ} \mathrm{C}$ for $15 \mathrm{sec}$ and $55^{\circ} \mathrm{C}$ for $45 \mathrm{sec}$. HAND2-AS1 expression was normalized to $\beta$-actin using the $2^{-\Delta \Delta \mathrm{Cq}}$ method (9). The experiment was performed in triplicate.

Cell proliferation assay. A 96-well plate was seeded at $4 \times 10^{3}$ cells/well and cultured at $37^{\circ} \mathrm{C}\left(5 \% \mathrm{CO}_{2}\right)$. At the $24-, 48-$, 72- and 96-h time points, $10 \mu \mathrm{l}$ Cell Counting Kit-8 (CCK-8) solution was added to each well; cells were cultured for a further $4 \mathrm{~h}$, and OD values were measured at $450 \mathrm{~nm}$ using the Fisherbrand $^{\mathrm{TM}}$ accuSkan $^{\mathrm{TM}}$ GO UV/Vis microplate spectrophotometer (Thermo Fisher Scientific, Inc.). The experiment was performed in triplicate.

Western blot analysis. Radioimmunoprecipitation assay buffer (Thermo Fisher Scientific, Inc.) was used to extract the total protein from cells, and the bicinchoninic acid assay method was used to measure protein concentration. Proteins were separated using SDS-PAGE with a $10 \%$ gel (30 $\mu \mathrm{g}$ protein/lane), and transferred to PVDF membranes. The membranes were blocked with $5 \%$ skimmed milk in PBS at room temperature for $1 \mathrm{~h}$, followed by incubation with the following primary antibodies overnight at $4^{\circ} \mathrm{C}$ : Rabbit anti-glucose transporter 1 (GLUT1; 1:2,000; cat. no. ab15309) and anti-GAPDH (1:1,000; ab8245; all Abcam, Cambridge, UK). The following day, the membranes were 

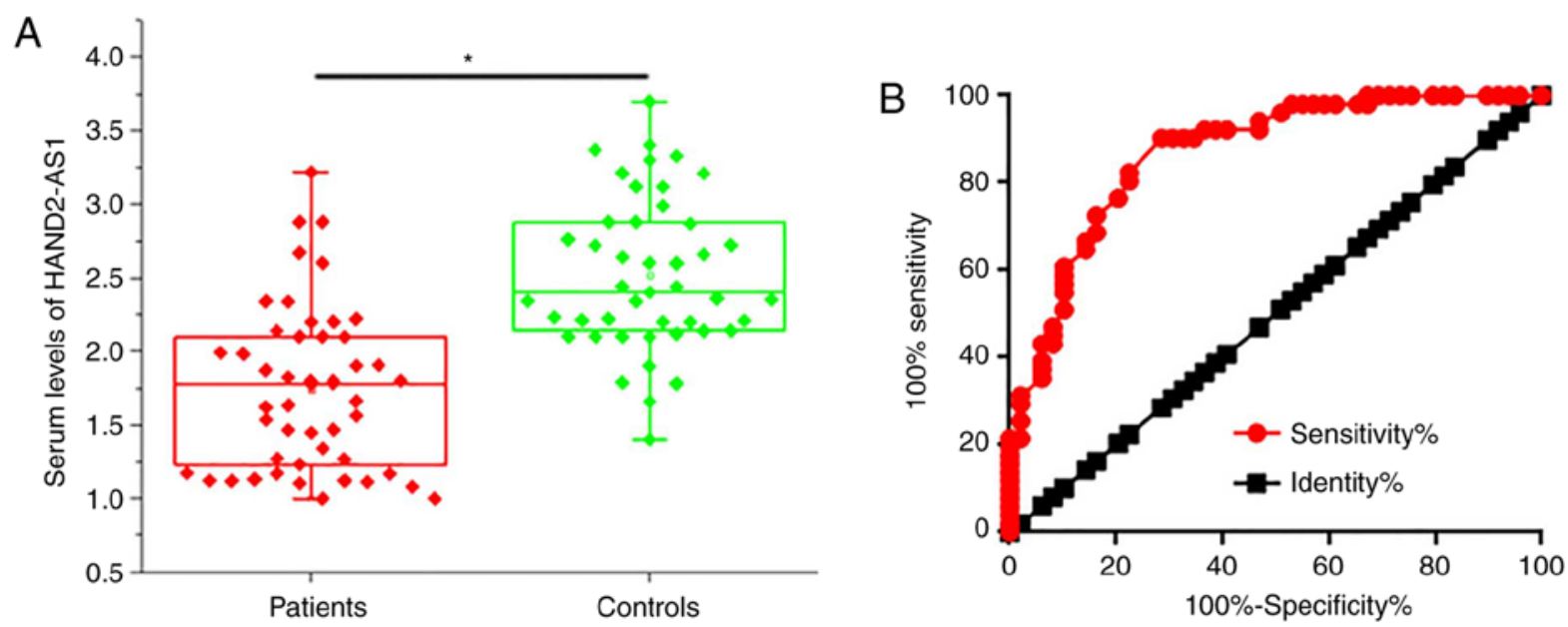

Figure 2. Comparison and diagnostic values of serum levels of HAND2-AS1 between patients with osteosarcoma and healthy controls. Serum levels of HAND2-AS1 were measured using reverse transcription-quantitative polymerase chain reaction. (A) Comparison of serum levels of HAND2-AS1 between osteosarcoma patients and healthy controls. Serum levels of HAND2-AS1 were significantly higher in the controls compared with those in osteosarcoma patients. (B) Receiver operating characteristic curve of HAND2-AS1 in the diagnosis of osteosarcoma. Downregulation of HAND2-AS1 effectively distinguished osteosarcoma patients from healthy controls. "P<0.05. n=3. HAND2-AS1, heart and neural crest derivatives expressed 2-antisense RNA 1.

incubated with horseradish peroxidase-conjugated secondary antibody (1:1,000; cat. no. MBS435036; MyBioSource, Inc.) for $1 \mathrm{~h}$ at room temperature. Protein bands were visualized using an Enhanced Chemiluminescence detection reagent (Sigma-Aldrich; Merck KGaA), and Image J software, v1.8.0 (National Institutes of Health) was used to normalize the expression levels of GLUT1 to the endogenous $\beta$-actin control. The experiment was performed in triplicate.

Statistical analysis. SPSS software version 19.0 (IBM Corp.) was used for all statistical analyses. Data are expressed as the mean \pm standard deviation. Comparisons between two groups and among multiple groups were performed using the Student's t-test and one-way analysis of variance, followed by the Tukey's test, respectively. Count data were analyzed using the $\chi^{2}$ test. Receiver operating characteristic (ROC) curve analysis was performed to evaluate the diagnostic value of HAND2-AS1 serum levels in osteosarcoma, with patients as true-positive samples and healthy controls as true-negative samples. $\mathrm{P}<0.05$ was considered to indicate a statistically significant difference.

\section{Results}

Expression of HAND2-AS1 in the tumor tissues and adjacent healthy tissues of 48 patients with osteosarcoma. Expression levels of HAND2-AS1 in the osteosarcoma tissues and healthy tissues of 48 patients were measured using RT-qPCR. As illustrated in Fig. 1, expression levels of HAND2-AS1 were significantly lower in osteosarcoma tissues compared with those in the adjacent tissues in 41 of the 48 patients $(\mathrm{P}<0.05)$. Only 3 patients displayed significantly lower expression levels of HAND2-AS1 in adjacent tissues compared with those in osteosarcoma tissues $(\mathrm{P}<0.05)$. No significant differences were observed in the remaining 4 patients.

Comparison and diagnostic values of HAND2-AS1 serum levels between patients with osteosarcoma and healthy controls. Serum levels of HAND2-AS1 were determined using
RT-qPCR. Serum levels of HAND2-AS1 were significantly higher in control subjects compared with those in osteosarcoma patients $(\mathrm{P}<0.05$; Fig. 2A). ROC curve analysis was performed with osteosarcoma patients as true-positive and healthy controls as true-negative samples. The area under the curve (AUC) was 0.8685 , with a $95 \%$ confidence interval of 0.7989-0.9382 ( $\mathrm{P}<0.0001$; Fig. 2B). Therefore, serum HAND2-AS1 level may hold potential diagnostic value in osteosarcoma.

Correlation between serum levels of HAND2-AS1 and clinicopathological features. Patients were divided into high and low expression groups according to the median serum level of HAND2-AS1. The $\chi^{2}$ test was performed to analyze correlations between serum levels of HAND2-AS1 and clinicopathological patient data. As displayed in Table I, there were no significant correlations between the serum levels of HAND2-AS1 and patients' age, sex and distant tumor metastasis. However, a significant correlation was observed between serum levels of HAND2-AS1 and tumor size.

Effects of HAND2-AS1 siRNA silencing on glucose uptake. The data in Table I indicate that HAND2-AS1 is associated with tumor growth in osteosarcoma. Glucose metabolism is critical for the survival and proliferation of cancer cells (10). In the present study, the effects of HAND2-AS1 siRNA silencing on glucose uptake were investigated in osteosarcoma cells. HAND2-AS1 siRNA silencing significantly promoted glucose uptake in the human osteosarcoma MG-63 and SAOS-2 cell lines $(\mathrm{P}<0.05$; Fig. 3$)$, but not in the normal bone hFOB cell line.

Effects of HAND2-AS1 siRNA silencing on GLUT1 expression. Compared with that in normal bone cells (hFOB), HAND2-AS1 expression was significantly downregulated (Fig. 4A), while GLUT1 protein was significantly upregulated (Fig. 4B), in the osteosarcoma MG-63 and SAOS-2 cell lines. HAND2-AS1 siRNA silencing led to significantly upregulated expression of GLUT1 in MG-63 and SAOS-2 cells $(\mathrm{P}<0.05)$, but not in hFOB cells (Fig. 4C). 
Table I. Correlation between serum levels of heart and neural crest derivatives expressed 2-antisense RNA 1 and the clinicopathological data of the patients.

\begin{tabular}{lcccccc}
\hline Variable & Group & Cases, $\mathrm{n}$ & High expression, $\mathrm{n}$ & Low expression, $\mathrm{n}$ & $\chi^{2}$ value & P-value \\
\hline Sex & Male & 28 & 12 & 16 & 1.37 & 0.24 \\
& Female & 20 & 12 & 8 & & \\
Age, years & $>30$ & 26 & 11 & 15 & 1.34 & 0.25 \\
\multirow{2}{*}{ Primary tumor diameter, cm } & $>30$ & 22 & 13 & 9 & & \\
& $>5$ & 23 & 17 & 18 & & 0.001 \\
Tumor distant metastasis & $\geq 5$ & 25 & 7 & 8 & 0.78 & 0.38 \\
& Yes & 19 & 11 & 16 & & \\
\end{tabular}

A
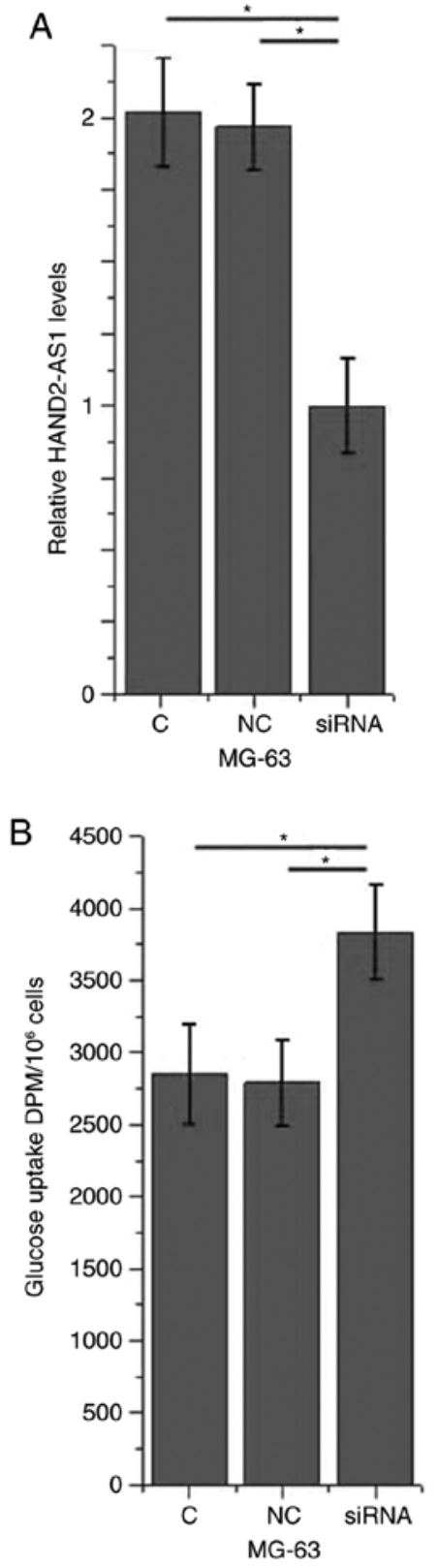
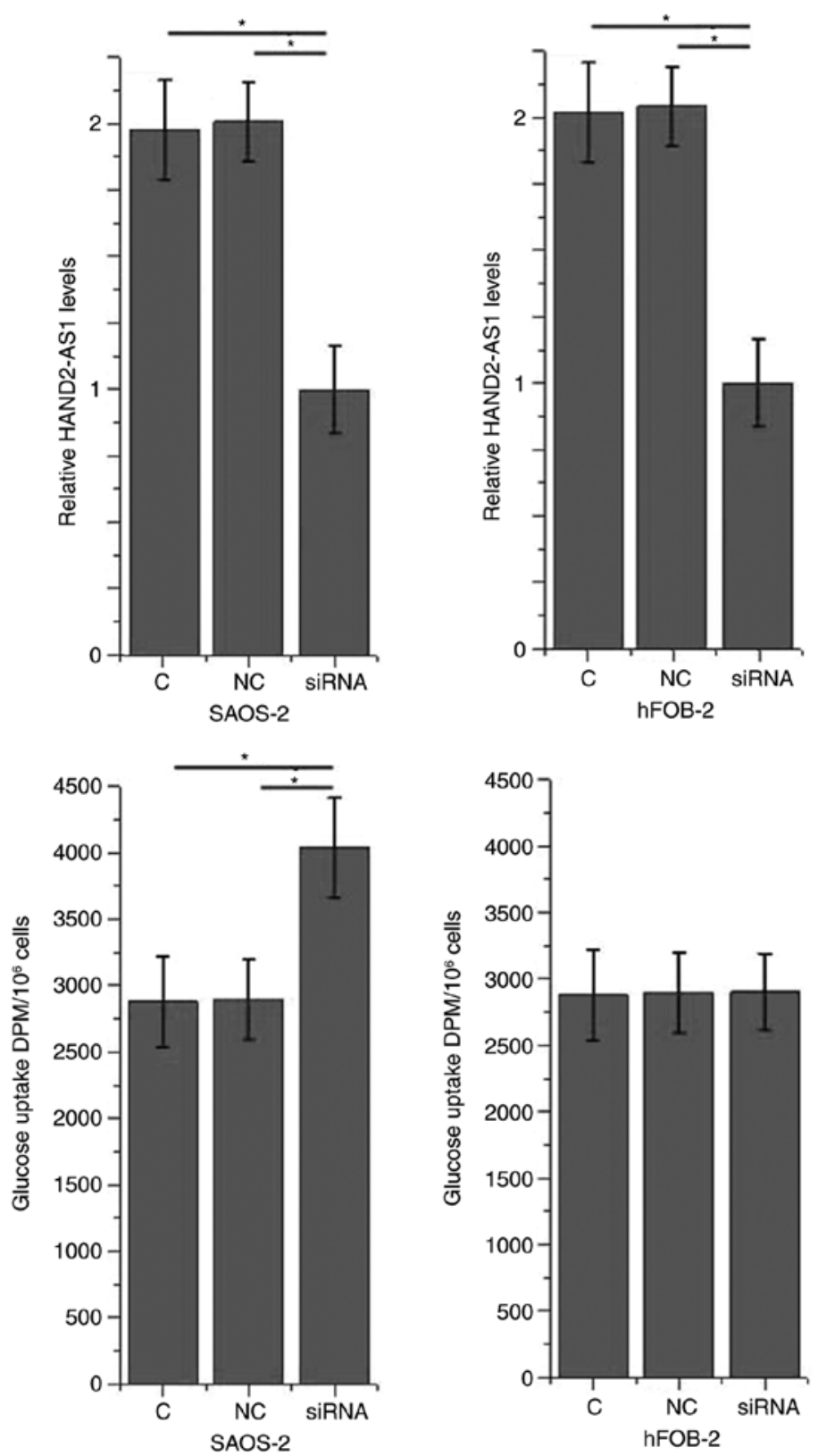

Figure 3. Effects of HAND2-AS1 siRNA silencing on glucose uptake. The effects of HAND2-AS1 siRNA silencing on glucose uptake in osteosarcoma cells were investigated by glucose uptake assay. (A) HAND2-AS1 siRNA silencing significantly reduced the levels of HAND2-AS1 in MG-63, SAOS-2 and hFOB cell lines, and (B) promoted glucose uptake in MG-63 and SAOS-2, but not hFOB cells. "P<0.05. n=3. HAND2-AS1, heart and neural crest derivatives expressed 2-antisense RNA 1; siRNA, small interfering RNA; C, control; NC, negative control. 


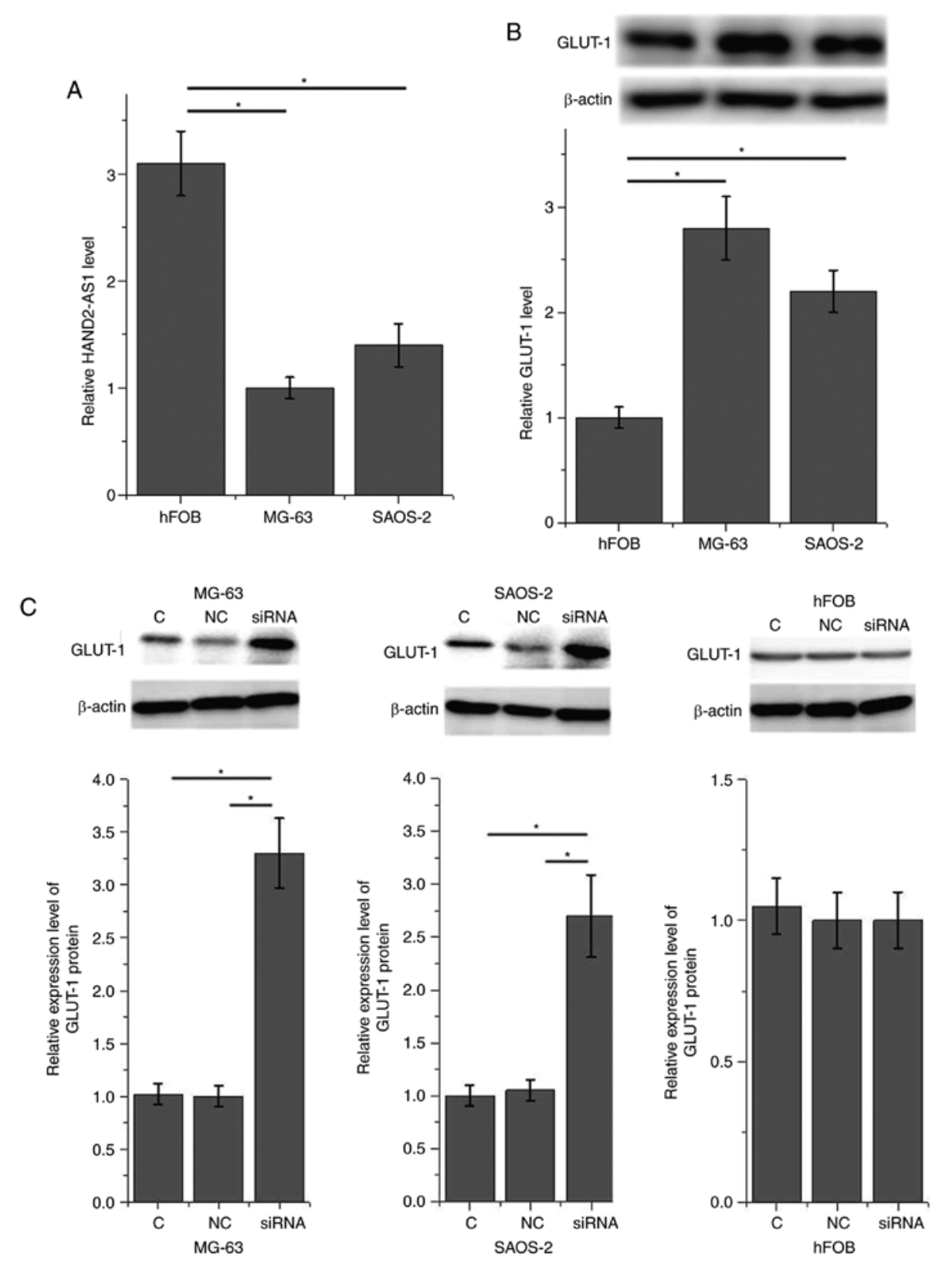

Figure 4. Effects of HAND2-AS1 siRNA silencing on GLUT1 expression. The effects of HAND2-AS1 siRNA silencing on GLUT1 expression were investigated by western blotting. Compared with normal bone hFOB cells, in MG-63 and SAOS-2 cells, (A) The non-coding RNA, HAND2-AS1 was significantly downregulated, while (B) GLUT1 expression was significantly upregulated. (C) HAND2-AS1 siRNA silencing led to significantly upregulated expression of GLUT1 in MG-63 and SAOS-2 cells, but not in hFOB cells. "P>0.05. n=3. HAND2-AS1, heart and neural crest derivatives expressed 2-antisense RNA 1; GLUT1, glucose transporter 1; siRNA, small interfering RNA; C, control; NC, negative control.
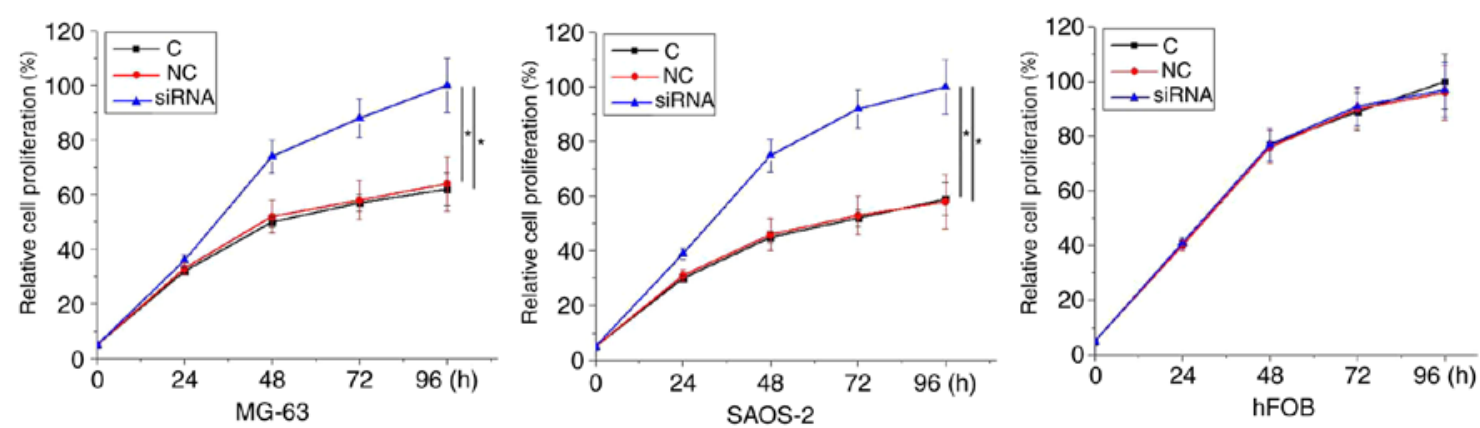

Figure 5. Effects of HAND2-AS1 siRNA silencing on cell proliferation. The effects of HAND2-AS1siRNA silencing on cell proliferation were investigated using the CCK-8 assay. HAND2-AS1 siRNA silencing significantly promoted the proliferation of human osteosarcoma MG-63 and SAOS-2 cell lines, but not cells of the normal bone $\mathrm{hFOB}$ cell line. ${ }^{*} \mathrm{P}>0.05 . \mathrm{n}=3$. HAND2-AS1, heart and neural crest derivatives expressed 2-antisense RNA 1; siRNA, small interfering RNA; C, control; NC, negative control. 
Effects of HAND2-AS1 siRNA silencing on cell proliferation. The proliferation rates of the 3 cell lines were detected using the CCK-8 assay. HAND2-AS1 siRNA silencing significantly promoted the proliferation of the human osteosarcoma MG-63 and SAOS-2 cell lines ( $\mathrm{P}<0.05$; Fig. 5), but not the cells of the normal bone cell line, hFOB.

\section{Discussion}

In the present study, a novel lncRNA with characterized functionality in endometrioid endometrial carcinoma, and the growth of osteosarcoma tumors, was reported. It was also observed that the action of this IncRNA in osteosarcoma is likely achieved by inhibiting osteosarcoma cell proliferation through disruption of glucose metabolism. This highlighted a potential therapeutic target for osteosarcoma.

The pathogenesis of osteosarcoma is influenced by numerous lncRNAs and their varied roles in the onset, development and progression of cancer. Upregulation of lncRNA-highly upregulated in liver cancer (HULC) was previously observed in osteosarcoma tissues compared with that in adjacent healthy tissues, and reducing the expression level of IncRNA HULC was suggested to be a potential treatment for osteosarcoma (11). Overexpression of nuclear paraspeckle assembly transcript 1 is involved in the development of drug resistance in osteosarcoma cells. Therefore, knockdown of this oncogenic lncRNA may improve the outcome of drug treatment in osteosarcoma (12). By contrast, growth arrest specific 5 is considered to be a tumor suppressor lncRNA and displays a downregulated expression pattern in osteosarcoma (13). lncRNA HAND2-AS1 is a recently identified lncRNA with decreased expression levels in endometrioid endometrial carcinoma tissues (7), indicating its potential role as a tumor suppressor in this disease. In the present study, significantly lower expression levels of lncRNA HAND2-AS1 in tumor tissues (compared with adjacent healthy tissues) was observed in the majority of patients with osteosarcoma, indicating its potential role as a tumor suppressor.

The survival rate of osteosarcoma patients with distant tumor metastasis is low, and an increase in the early diagnostic rate is required to improve treatment outcomes. The blood not only transports nutrients around the body, but also delivers signaling molecules, and the development of human diseases is usually accompanied by alterations in certain blood constituents $(14,15)$. Detecting these alterations in the blood may provide guidance for the treatment of human diseases $(16,17)$. In the present study it was observed that serum levels of lncRNA HAND2-AS1 were significantly lower in osteosarcoma patients compared with those in healthy controls. ROC curve analysis also revealed that serum HAND2-AS1 may be used to effectively distinguish osteosarcoma patients from healthy individuals. These data suggest that serum HAND2-AS1 may serve as a potential diagnostic marker for osteosarcoma. It is worth noting that HAND2-AS1 is a recently identified lncRNA with an unknown expression pattern in the majority of human diseases. Therefore, multiple markers may be used to improve diagnostic accuracy.

The present study revealed an association between HAND2-AS1 and tumor growth, but not metastasis. Glucose metabolism provides energy for all cell types, although abnormal glucose metabolism is a unique marker of cancerous cells (6). HAND2-AS1 siRNA silencing significantly promoted glucose uptake in 2 osteosarcoma cell lines, indicating that HAND2-AS1 may be an inhibitor of glucose uptake in osteosarcoma. As a key component of glucose metabolism, GLUT1 is ordinarily upregulated in cancer cells (18). HAND2-AS1 siRNA silencing significantly promoted the expression of GLUT1, and also promoted proliferation in 2 osteosarcoma cell lines. The data suggest that HAND2-AS1 may inhibit the proliferation of osteosarcoma cells by targeting glucose uptake through the downregulation of GLUT1.

Additionally, HAND2-AS1 siRNA silencing had no significant effects on cells of the normal bone hFOB cell line. Therefore, HAND2-AS1 may serve as a potential therapeutic target for osteosarcoma specifically.

In conclusion, HAND2-AS1 is downregulated in osteosarcoma and may inhibit the growth of osteosarcoma tumors through its interaction with glucose metabolism.

\section{Acknowledgements}

Not applicable.

\section{Availability of data and materials}

All data generated or analyzed during this study are included in this published article.

\section{Author's contributions}

$\mathrm{SC}, \mathrm{XX}, \mathrm{SL}$ and $\mathrm{BH}$ were responsible for the conception and design of the study. SC and XX performed the experiments. SC, XX, SL and BH analyzed and interpreted the data. SC and XX drafted the article. SC, XX, SL and BH were responsible for the revision of the manuscript. All authors read and approved the final manuscript.

\section{Ethics approval and consent to participate}

The protocols of the present study were approved by the Ethics Review Committee of Jingzhou Central Hospital (Jingzhou, China). All patients provided written informed consent.

\section{Patient consent for publication}

Not applicable.

\section{Competing interests}

The authors declare that they have no competing interests.

\section{References}

1. Isakoff MS, Bielack SS, Meltzer P and Gorlick R: Osteosarcoma: Current treatment and a collaborative pathway to success. J Clin Oncol 33: 3029-3035, 2015.

2. Botter SM, Neri D and Fuchs B: Recent advances in osteosarcoma. Curr Opin Pharmacol 16: 15-23, 2014.

3. Luetke A, Meyers PA, Lewis I and Juergens H: Osteosarcoma treatment-where do we stand? A state of the art review. Cancer Treat Rev 40: 523-532, 2014. 
4. Lu C and Jiang DL: Expression of POSTN mRNA is associated with osteosarcoma prognosis. Int J Clin Exp Pathol 9: 10664-10669, 2016

5. Li J, Liu L, Li J, Chen Y, Jiang XW, Ouyang YR, Liu YQ, Zhong H, Li H and Xiao T: Microarray expression profile of long noncoding RNAs in human osteosarcoma. Biochem Biophys Res Commun 433: 200-206, 2013.

6. Hay N: Reprogramming glucose metabolism in cancer: Can it be exploited for cancer therapy? Nat Rev Cancer 16: 635-649, 2016.

7. Yang X, Wang CC, Lee WYW, Trovik J, Chung TKH and Kwong J: Long non-coding RNA HAND2-AS1 inhibits invasion and metastasis in endometrioid endometrial carcinoma through inactivating neuromedin U. Cancer Lett 413: 23-34, 2018.

8. Zheng X, Han H, Liu GP, Ma YX, Pan RL, Sang LJ, Li RH, Yang LJ, Marks JR, Wang W and Lin A: LncRNA wires up Hippo and Hedgehog signaling to reprogramme glucose metabolism. EMBO J 36: 3325-3335, 2017.

9. Livak KJ and Schmittgen TD: Analysis of relative gene expression data using real-time quantitative PCR and the 2(-Delta Delta C(T)) method. Methods 25: 402-408, 2001

10. Boroughs LK and DeBerardinis RJ: Metabolic pathways promoting cancer cell survival and growth. Nat Cell Biol 17: 351-359, 2015.

11. Kong D and Wang Y: Knockdown of lncRNA HULC inhibits proliferation, migration, invasion and promotes apoptosis by sponging miR-122 in osteosarcoma. J Cell Biochem 119: 1050-1061, 2018

12. Hu Y, Yang Q, Wang L, Wang S, Sun F, Xu D and Jiang J: Knockdown of the oncogene LncRNA NEAT1 restores the availability of miR-34c and improves the sensitivity to cisplatin in osteosarcoma. Biosci Rep 38: pii: BSR20180375, 2018.
13. Wang Y and Kong D: LncRNA GAS5 represses osteosarcoma cells growth and metastasis via sponging miR-203a. Cell Physiol Biochem 45: 844-855, 2018

14. Kawashima M, Yamamura M, Taniai M, Yamauchi $H$, Tanimoto T, Kurimoto M, Miyawaki S, Amano T, Takeuchi T and Makino H: Levels of interleukin-18 and its binding inhibitors in the blood circulation of patients with adult-onset Still's disease. Arthritis Rheum 44: 550-560, 2001.

15. Yaman Agaoglu F, Kovancilar M, Dizdar Y, Darendeliler E, Holdenrieder S, Dalay N and Gezer U: Investigation of miR-21, miR-141 and miR-221 in blood circulation of patients with prostate cancer. Tumour Biol 32: 583-588, 2011.

16. Sabbisetti VS, Waikar SS, Antoine DJ, Smiles A, Wang C, Ravisankar A, Ito K, Sharma S, Ramadesikan S, Lee M, et al: Blood kidney injury molecule-1 is a biomarker of acute and chronic kidney injury and predicts progression to ESRD in type I diabetes. J Am Soc Nephrol 25: 2177-2186, 2014.

17. Zhao M, Zhou Y, Zhu B, Wan M, Jiang T, Tan Q, Liu Y, Jiang J, Luo S, Tan Y, et al: IFI44L promoter methylation as a blood biomarker for systemic lupus erythematosus. Ann Rheum Dis 75: 1998-2006, 2016.

18. Kunkel M, Reichert TE, Benz P, Lehr HA, Jeong JH, Wieand S, Bartenstein P, Wagner W and Whiteside TL: Overexpression of Glut-1 and increased glucose metabolism in tumors are associated with a poor prognosis in patients with oral squamous cell carcinoma. Cancer 97: 1015-1024, 2003.

This work is licensed under a Creative Commons Attribution-NonCommercial-NoDerivatives 4.0 International (CC BY-NC-ND 4.0) License. 